\title{
recp| $\begin{aligned} & \text { Revista Eletrônica } \\ & \text { de Ciência Política }\end{aligned}$
}

EXPEDIENTE

Vol. 5, n. 2, 2014.

ISSN 2236-451X

Editor-sênior / coordenador do PPGCP-UFPR: Emerson Urizzi Cervi

\section{Editores-Executivos}

José Augusto Hartmann; Karolina Mattos Roeder; Márcio Cunha Carlomagno

\section{Comitê Editorial}

Diego Silveira Coelho Ferreira; Eric Gil Dantas; Flávia Bozza Martins; Isabele Batista Mitozo; Jaqueline da Silva Borges; Jaqueline Kleine Buckstegge; José Augusto Hartmann; Larissa Bozza Isquierdo; Karolina Mattos Roeder; Márcio Cunha Carlomagno; María Alejandra Nicolás; Paula Matoski Butture.

Revisão de texto: Isabele Batista Mitozo; Jaqueline Kleine Buckstegge; Karolina Mattos Roeder; Márcio Cunha Carlomagno.

Capa e diagramação: Márcio Cunha Carlomagno

\section{Conselho Consultivo}

Dr. Adriano Nervo Codato (UFPR), Alexsandro Eugenio Pereira (UFPR), Álvaro Gabriel Bianchi (UNICAMP), Armando Boito Jr. (UNICAMP), Bruno Bolognesi (UNILA), Emerson Cervi (UFPR), Fabrício Tomio (UFPR), Flávio da Cunha Rezende (UFPE), Helcimara Telles (UFMG), Giovana Bonamim (Instituto de Desarrollo Económico y Social, IDES - Argentina), Gonzalo Adrián Rojas (UFCG), Gustavo Biscaia de Lacerda (UFPR), Huascar Fialho Pessali (UFPR), Icaro Engler (UFRGS), João Feres Júnior (IESP-UERJ) Luciana Veiga (UFPR), Marcus André Melo (UFPE), Maria do Socorro Souza Braga (UFSCar) Mário Fuks (UFMG), Marta Teresa da Silva Arretche (USP), Michele Goulart Massuchin (UFSCar), Nelson Rosário de Souza (UFPR), Paulo Roberto Neves Costa (UFPR), Raquel Kritsch (USP-UEL) Renato Perissinotto (UFPR), Rogério Bastos Arantes (USP), Rodrigo Rossi Horochovski (UFPR), Samira Kauchakje (PUC-PR e UFPR), Sérgio Braga (UFPR). 